\title{
Humanizing the Localizing Sustainable Development Goals (SDGs) in Education and Research at Higher Education Institutions (HEls)
}

\author{
Mohd Fadhil Md Din ${ }^{1, *}$, Wahid Omar ${ }^{2}$, Shazwin Taib ${ }^{3}$, Shamsul Sarip ${ }^{4}$, Santhana Krishnan ${ }^{5}$ \\ 1,2,3,4,5 Universiti Teknologi Malaysia, 81310 Johor Bahru, Johor, Malaysia \\ *corresponding author: mfadhil@utm.my
}

\section{Article Info}

Received:

15 March 2021

Accepted:

25 May 2021

Published:

1 August 2021

DOI:

Presented in The $6^{\text {th }}$ International (Virtual) Workshop on UI GreenMetric World University Rankings (IWGM 2020)
Abstract Preliminary actions taken by the Universiti Teknologi Malaysia (UTM) towards the value-added from the Sustainable Development Goals (SDGs) framework that may relevant in the current indicators, elements, values and impacts, and the also constructive analysis by different sectors at High Educational Institutions (HEls). Typically, the instrumentation on performances of each element (or indicator) in research and education portfolios are majorly defined as Key Performance Index (KPI) and/or Key Achievement/Amal Index (KAls). All of instrumentations suitable at the vertical strategic plan to disseminate the workload across stakeholders in the HEls. A strategic plan by each university will represent the core values and relevancy of niche area in academic and research sectors. However, UTM already is identifying the most critical element, far beyond the $\mathrm{KPI} / \mathrm{KAl}$ successful story which focusses under the Pelan Global Universiti (PGU I-III) to outstanding values of inclusiveness, synergy and visibility with the important Desired State 2020. The UTM core values will serve Integrity, Synergy, Excellent and Sustainability (ISES) in all operation/services throughout trustworthy engagement with stakeholders. Meantime, the action plan for enVision 2025 institualized the pragmatic roadmaps align with the SDGs at the global benchmark, in order to horizontally manage the great grassroots improvement and further develop unique vision to UTM; named as UTMDNA. This paper describes the proactive strategic plan by UTM management to advocate the next roadmap altogether with high impact sustainable education and research works. Most of the concurrent achievement, action-plan and long-term Industrial Revolution 4.0 (IR 4.0) is emphasized in the life-balance strategy framework. The second wave (2020 - 2030) of SDGs in UTM is among the most recent thoughts towards the sustainability requirements specially to serve the UN SDG and enVision 2025 (UTM). Therefore, Malaysian higher education institutions should take proactive steps in culturing SDG initiatives - guided but not bounded by the specific measures set-out in the UN Conference.

\section{Keyword:}

Localizing SDGs, HEls, Pelan Global Universiti, Higher Education Institutions 


\section{Introduction to the Governance of Sustainability at University}

Campus sustainability for higher education institutions (HEIs) involves minimizing negative impact of its activity on the environment and society, which usually covers the common pillars in the sustainability action plan. This approach promotes a balanced environment, economy and society in a more structured way in terms of prudent use of resources to meet the function of teaching, research, operational and community can be responsible for the sustainability init.iatives in an academic institutions, which can act as an impetus in sustaining the momentum and facilitating the process of achieving a sustainable campus [1]. The most suitable method to regulate the excercises is through the Living Laboratory (LL) and utmost delivering the action-plan, strategic overview and strong supports from different stakeholders within the campus governance and operation. Meanwhile, the strong support governance support from UTM is via the establishment of UTM Campus Sustainability, which revisit all the sustainable component in campus, include:

a. UTMCS functions as a facilitator for campus sustainability initiatives led by key internal stakeholders. It also bridges the gap between research and operations by implementing more research outputs on campus. In implementing the Green Offices initiative, UTMCS works closely with technical experts to develop co-management of Campus Sustainability initiatives, comprising the Sustainable Energy Management Committee, JHB (Jabatan Harta Bina) and the Registrar's Office, and many more taskforces

b. Ongoing Campus Sustainability campaigns are conducted with the support of the student representative council, aimed at fostering a culture of sustainability and assisting the campus to achieve climate neutrality.

c. External engagements, attending local and international conferences and workshops related to sustainability are among the strategies to accelerate the campus sustainability process to meet national and global standards.

\subsection{SDG Localization Ideas and Implementation Impact}

The latest approaches to ensure a holistic implementation of SDGs at the University level include the "Think Global! Act Locally!" and "Think Long-term! Act Now!" concepts [2]. In UTM, the hastag provided to synergize the implementation including \#UTMDNA, \#UTMSDGs, and \#LocalSDGs. SDG related transformational agenda are generally misinterpreted as programs driven by political agenda, involved limited community participation at the policy level, guided by unreasonable targets and peppered with implementation issues. In addition, the lack of consistent data collection in some countries remains a major challenge for scientific input to effectively address these challenges. The confusion among grassroots and stakeholders have been addressed through the introduction of the "Localizing SDGs" initiative (known as Localization SDGs). The initiatives introduced are not new programs and remain within the SDG contexts but are re-aligned to local strategies for managing and implementing SDGs based on socio-cultural, economic and lifestyle activities. For example, some of the developing countries do not require technology as a prerequisite for the implementation of the indicators / elements within SDGs. Instead implementation initiatives and transformations at the community level are required to meet local socio-economic needs. Finally, these indicators remain inter-dependent on value change, cumulative impact and contribution of each SDG element.

The same is being developed through the involvement of higher education institutions, and this is true of Universiti Teknologi Malaysia (UTM). The process of refining and 
integrating the SDGs at UTM campus not only focused on justifying the development of environmental partnerships but also incorporating the framework in the University Global Plan (I-III) and involving all stakeholders within the campus (Figure 1). UTM is assessing and debating the needs of the Key Focus Area (KFA) which incorporates university strategic plans that build on short-term needs in terms of organizational structure and empowerment. Each year, the university will re-examine the initiatives undertaken, especially involving quantitative value, impact, and significance based on the current developments. These include policy, education, research, operation, community and financial structure, which include several procedures measured through the Key Amal Indicators (KAIs) index. Unlike the 2015 experiences, the inclusive approach to SDGs in the university strategic planning will reinforce the medium and long-term needs, especially involving governance, finance and operation. Preliminary actions taken by UTM add value to the strategic plan by using the SDGs framework throughout the selection of indicators, elements, values and impacts, and the constructive analysis by different sectors. Therefore, Malaysian higher education institutions should take proactive steps in culturing SDG initiatives - guided but not bounded by the specific measures set-out in the UN Conference.

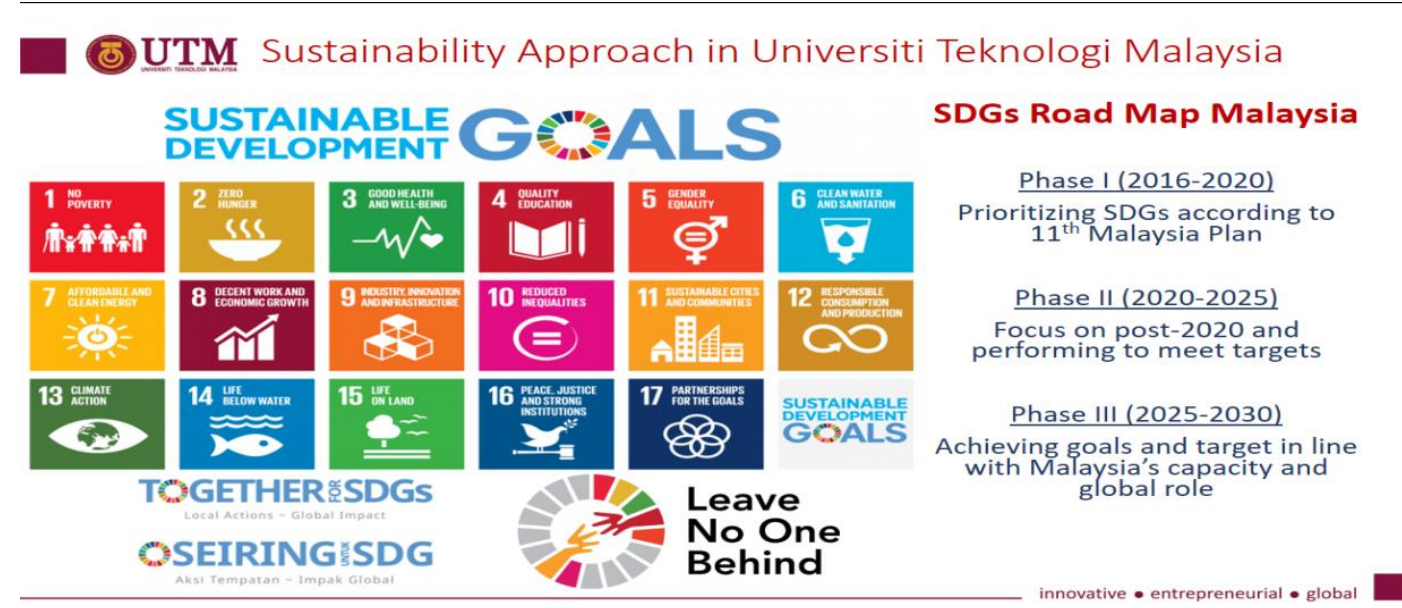

Figure 1: UTM SDGs governance roadmap towards the national blueprint

\subsection{Readiness and Preparation for SDG Localisation}

The second wave (2020 - 2030) of SDGs in UTM is among the most recent thoughts towards meeting sustainability requirements in the education system and administration. Based on the TWI 2050 redefinition, captured from the published elsewhere, the planning framework opens-up opportunities for any university-level initiatives to meet the established indicators are known as the Low Carbon Campus (LCC) Plan Initiative. UTM already developed the few options in LCC since 2015, and the current aims towards Zero and/or Net Carbon campus. Various instruments have been used to evaluate the effectiveness of the implementation especially in the energy and infrastructure sectors, which useful in determining the original works from the past experience. In the past, measurements using the Low Carbon City Framework (LCCF) and MyCarbon databases have helped gauge the energy sector's contribution to carbon reduction and energy-saving programs on campus, as the result in the Car-Free Campus initiatives. Another instrument introduced by the MGTC (Malaysia Green Tech Corporation) is the Building Consumption Input System (BCiS) which is among the advances made to ensure consistent data collection at various levels in the public or government sector and further enhancing the attainment of 
the Decarbonization Community.

These instruments are fundamental to the data collection process and require little change in terms of mentality-attitude in the KPI-based instrumentation. Based on previous experiences and campaigns implemented over the years, UTM Sustainable Campus has identified several key initiatives that can be realised through the Living Laboratory. Thus, a single instrument incorporating all elements of the SDGs was included in the rating system known as UTM GreenLeaf ${ }^{\circledR}$ and are categorized based on Office / Division / Unit, Residential College, and Faculty / School. The incorporation of these rating systems has enabled the adjustment of instruments at the university level through the PMI (Pool-MonitoringImprovement) method. In addition, through the UTM Envision 2020-2025 plan, a preparation phase will be implemented using the SDGs governance structures. One of the initial phases will involve utilising the SDG-based indicator policy for all administrative and service sectors to assist global reporting such as the Global Report Initiative (GRI) and THE Impact Rankings - SDGs. The next phase is to build an unbiased structural program involving all stakeholders who are not solely focused on the environment. Various initiatives have been planned under UTM Sustainable Campus Framework's High Impact Program throughout 2020 to ensure the involvement of all UTM community, leaving no one behind. The initiatives will cover three main foci, namely (1) enhancing the localisation of SDGs on campus; (2) implementing the Zero Waste Society concept; and (3) embedding elements of SDGs in sustainability programs. Therefore, UTM is one of the HEls in Malaysia that are truly serious about sustainability should show commitment in advocating SDG related initiatives not only as a sustainability benchmark but as a distinct hallmark of a world class institution (Figure 3)

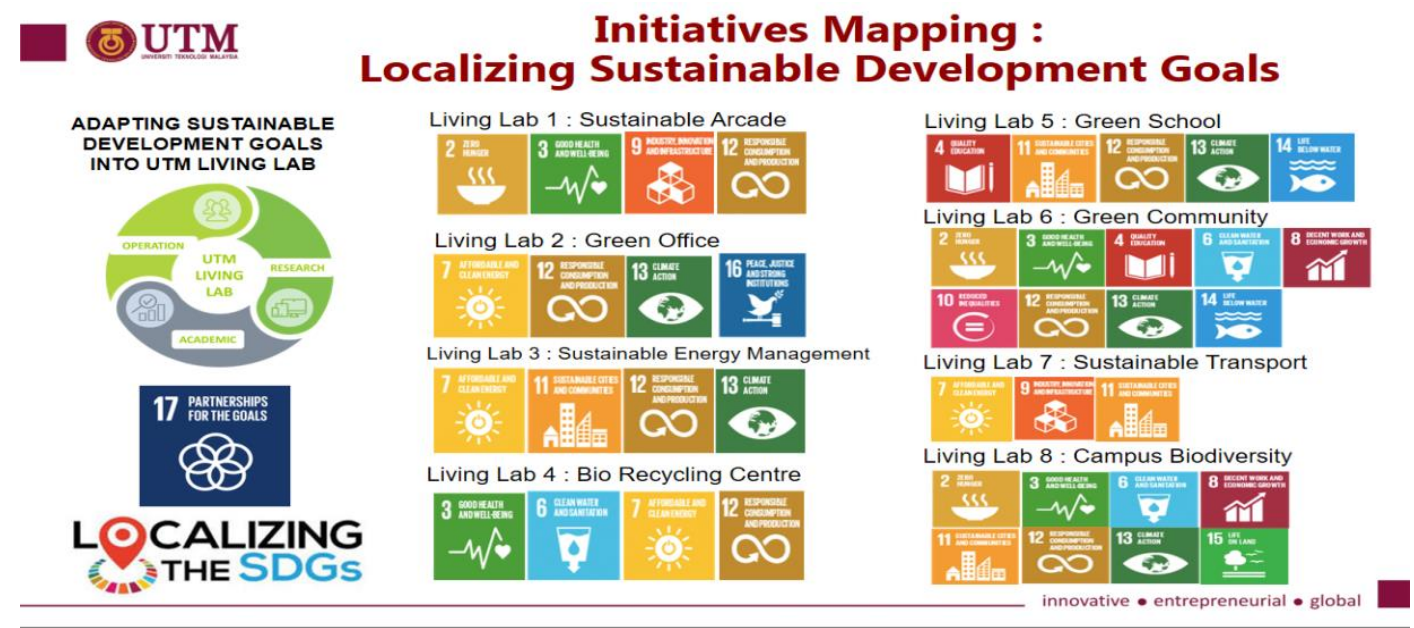

Figure 2: Localizing SDGs throughout the Living Laboratory

\section{Reality and Commitment from the SDGs Roadmap}

The idea of SDGs Roadmap and Action Plan for UTM engages few tools, missions, and readiness materials from the existing Plan Global and envision 2025. Part of them is Living Laboratory UTM (LL) and UTM Showcase (campus experience, products, and high impact program), as explained during the second wave (2020 - 2030) execution program at the university level. There are few suggestions of the existing Envision 2025, as follows:

\subsection{Administration and Governance}

a. Revision of UTM Policy based on the existing documentation embedded with SDGs 
b. Implementation of NEW Guidelines in UTM towards The World in 2050 in TWI2050 report [4] (link: https://www.unsdsn.org/news/2018/07/13/twi2050-reporttransformations-to-achieve-the-sustainable-development-goals)

- Human capacity

- Responsible consumption and Production (SDG-12)

- Decarbonize Energy System

- Accessing Nutritious Food and Clean Water

- Transformation cities/campus (smart and efficient infrastructure)

- Science, Technology and Innovation (STI)

c. Strong development in Energizing Partnership (SDG-17) in all interconnected department/unit/division The World in 2050 (TWI2050) was established by SDSN Partner, International Institute for Applied Systems Analysis (IIASA) to provide scientific foundations for the 2030 Agenda. It is based on the voluntary and collaborative effort of more than 60 authors from about 20 institutions, and some 100 independent experts from academia, business, government, intergovernmental and non-governmental organizations from all the regions of the world, who met three times at IIASA to develop pathways toward achieving the SDGs. Presentations of the TWI2050 approach and work have been shown at many international meetings including the United Nations Science, Technology and Innovation Forums and the United Nations High-level Political Forums. Two important meetings were held, one focusing on governance organized by the German Development Institute (DIE) in Bonn, Germany and the other on regional perspectives organized by the Stockholm Resilience Centre (SRC) held in Kigali, Rwanda. This report examines the current trends and dynamics that promote and jeopardize the achievement of the SDGs. It presents the TWI2050 framework, the integrated pathways which harness the synergies and multiple benefits across SDGs, and approaches to governing this sustainability transformation. TWI2050 identifies six exemplary transformations which will allow achieving the SDGs and long-term sustainability to 2050 and beyond [3].

\subsection{Operation, Services and Deliveries}

a. All BSCs and Strategic Planning must consist of SDG-4, 9, 11 and 12 as the most strategic improvement in the facility and empowerment to the University's stakeholders.

b. Sub-transformation on Decarbonize Campus Initiatives (Zero Emission) Transportation, Energy, Waste and Buildings.

\subsection{Student Empowerment}

a. Youth Program Education Hub under SDSN Youth (link: https://www.unsdsn.org/youth). SDSN Youth educates young people about the Sustainable Development Goals and provides opportunities for them to pioneer innovative solutions to address the world's biggest challenges. With this aim, we create platforms for young people to connect, collaborate and integrate their ideas and perspectives into national and regional pathways for the implementation of the SDGs. The SDG Students Program creates spaces on universities where students with no prior experience or engagements with the SDGs can come together to learn about, 
engage with, and take action on the SDGs. The program aims to groom the average student into a supporter of the SDGs, such that they will carry the importance of sustainability into their future work upon graduation. The semi-physical, semi-virtual space will be hosted at local universities and Facebook Workplace, where Hub Members can connect and interact with others from around the world, as well as with SDSN Youth stakeholders from different programs.

b. Eco School for Student Empowerment (link: https://www.ecoschools.global/howdoes-it-work). The Eco-Schools program is an ideal way for schools to embark on a meaningful path towards improving the environmental footprint of a school, a change which inevitably leads to a more sustainable, less costly and more responsible school environment. Eco-Schools challenges students to engage in tackling environmental problems at a level where they can see tangible results, spurring them on to realise that they really can make a difference. Eco-Schools facilitates contact between participating institutions not just at the national level, but also internationally. These links provide an opportunity for schools to share environmental information, they can also be used as a means for cultural exchanges and for improving language skills.

\subsection{Research, Teaching and Innovation}

a. Society and Youth Agenda

- Youth Southeast ASEAN Leadership Initiatives (link: https://asean.usmission.gov/yseali/). The Young Southeast Asian Leaders Initiative (YSEALI) builds the leadership capabilities of youth in the region and promotes crossborder cooperation to solve regional and global challenges.

- eMPowering Youth ASEAN Program link: https://www.aseanfoundation.org/apply now 201920 empowering youths across asean is receiving applications). ASEAN Foundation is an organization from and for the people of ASEAN. Through various projects under the thematic areas of Arts and Culture, Community Building, Education and Media, the ASEAN Foundation empowers the people of ASEAN to contribute to the building of the ASEAN Community.

b. University Partnership Program under SDG Academy (link: https://www.unsdsn.org/university-partnership-program) - especially interdepartment, office, schools and faculty in single entity for teaching and training under sustainability. The SDG Academy Community of Practice invites global higher education institutions, NGOs, for-profit businesses, and relevant government entities to join a diverse community dedicated to advancing education for sustainable development through peer learning and the sharing of best practices, customized resource development, and opportunities for research and thought leadership.

\subsection{Community Engagement and Industrial Linkages}

a. Supporting Data Monitoring in SDGs Indicator and Implementation (Internal and External Networks) - based on translational project within national or region (link: https://www.sdsntrends.org/projects) - TRENDS (must co-partners with members). TReNDS supports a range of projects at global, national, and sub-national levels that explore and document issues relating to SDG monitoring, evolving data governance, and new data-sharing policy and practice standards. 
SDG localization master plan integration process is given in the Figure 3 . The UTM Global Plan 2012-2020 (PGU) has three phases in making UTM a renowned university in science, technology and engineering globally. PGU I and PGU II have been successfully implemented and achieved the desired goals, and now its quest to become the nation's top educational institution that excels in science, technology and engineering, and forging ahead in terms of global rankings with the development of the UTM Global Plan Phase III (20182020). The six main focus areas in PGU III are translated into Strategic Objectives (SO) and Key Amal Indicators (KAl) of various initiatives and programs to be implemented [5]. The success of PGU Phase III is dependent on four core values: Integrity, Synergy, Excellence and Sustainability (ISES) towards universal well-being that strives for the good of the stakeholders, mainly the community and industry, which are also introduced in PGU Phase III. Interestingly, UTMCS already established the Local SDGs indicator based on this elements and high impact program, which never exists before, [6]

The Local SDGs workshop involved the top management of UTM, the Associate Director of the stakeholders, all the Senior Directors and Directors, all Deans of Research Alliances and Head of Service Centre (PTJ) and Steering Committee to obtain input and views holistically on the strategies, programmes and actions to be implemented [7].

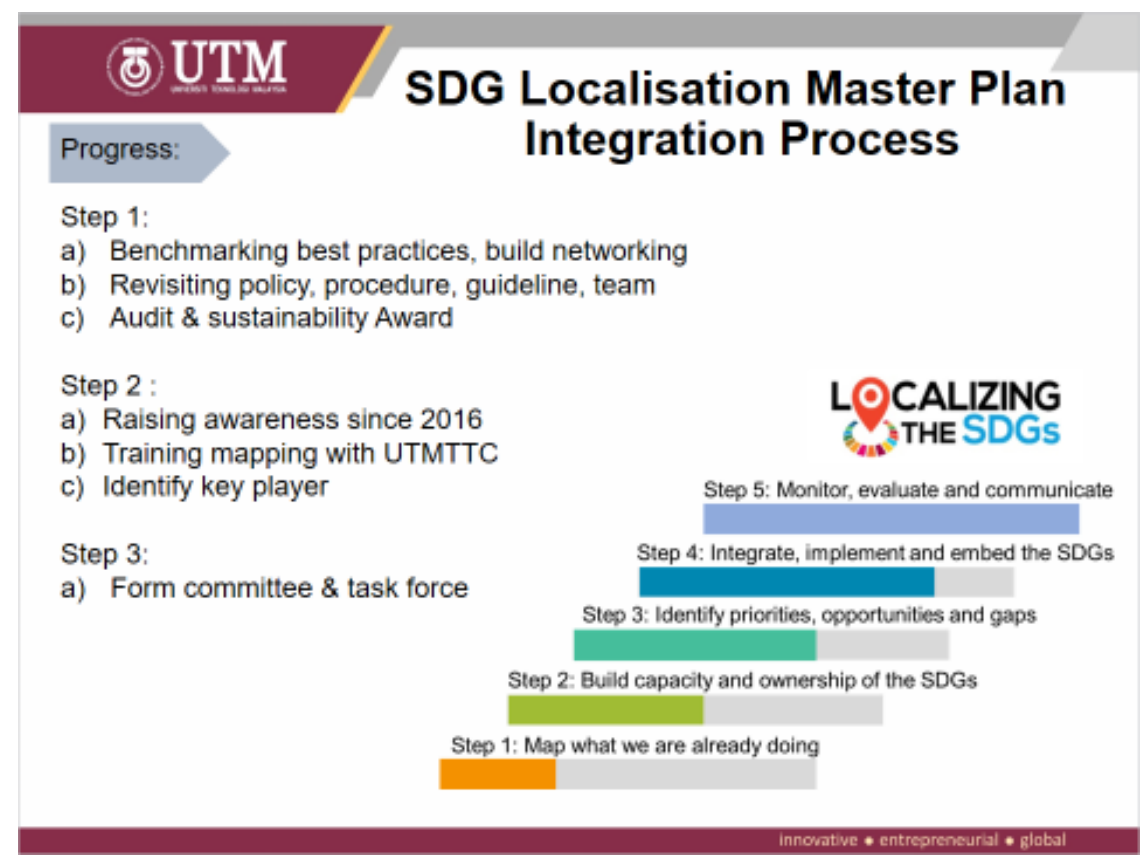

Figure 3: Localizing SDGs Masterplan Progress in UTM

\section{Summary/ Concluding Remarks}

Campus sustainability for higher education institutions (HEIs) approach promotes a balanced environment, economy and society in a more structured way in terms of prudent use of resources to meet the function of teaching, research, operational and community can be responsible for the sustainability init.iatives in an academic institutions, which can act as an impetus in sustaining the momentum and facilitating the process of achieving a sustainable campus. The latest approaches to ensure a holistic implementation of SDGs at the University level include the "Think Global! Act Locally!" and "Think Long-term! Act Now!" concepts. The same is being developed through the involvement of higher education institutions, and this is true of Universiti Teknologi Malaysia (UTM). The idea of SDGs 
Roadmap and Action Plan for UTM engages few tools, missions, and readiness materials from the existing Plan Global and envision 2025. Part of them is Living Laboratory UTM (LL) and UTM Showcase (campus experience, products, and high impact program), as explained during the second wave (2020 - 2030) execution program at the university level. Based on the TWI 2050 redefinition, captured from the published elsewhere, the planning framework opens-up opportunities for any university-level initiatives to meet the established indicators are known as the Low Carbon Campus (LCC) Plan Initiative. UTM already developed the few options in LCC since 2015, and the current aims towards Zero and/or Net Carbon campus. Various initiatives have been planned under UTM Sustainable Campus Framework's High Impact Program throughout 2020 to ensure the involvement of all UTM community, leaving no one behind.

\section{References}

1. Maric, P.F., Meier, S.K., 1992. Theoretical Methods in Higher Education Institution. Education Journal, Volume 83-87, pp. 119-128.

2. Green, M.A., 1987. High Efficiency in Higher Education Institution. University Publications, Switzerland.

3. Mishing, Y., 2004. Learning Processes in Developing Sustainable Campus, edited by D. Gupta Noyes Publications/William Andrew Publising, Norwich, NY, in press.

4. TWI2050 Report., 2018. Transformations to achieve the Sustainable Development Goals. Sustainable Development solutions Network. United Nations.

5. Ong, J.T., Dawley, P.G., 2013. High Efficiency of Wastewater Treatment. In: The Proceedings of the $1^{\text {st }}$ International Workshop on UI GreenMetric. Universitas Indonesia .

6. Clem, M., Rodriguez, J.A., Ashley, C.S., 2001. Annual Report of Sustanability Campus. Name of University.

7. UI GreenMetric, 2014. UI GreenMetric World University ranking. Available online at http://greenmetric.ui.ac.id/, accessed on date, month, year. 